\title{
Performance of new Load Balancing protocol for VANET using AODV [LBV_AODV]
}

\author{
R.K.Chauhan, Ph.D \\ Professor,Department Of Computer Science And \\ Applications,Kurukshetra University,Kurukshetra.
}

\author{
Arzoo Dahiya \\ ResearchScholar, \\ Department Of Computer Science And \\ application.Kurukshetra University.
}

\begin{abstract}
Vehicular Adhoc network has gained popular attention in last few years due to the provision of safety and comfort related applications.VANETs are direct offshoot of MANETs but with unique characteristics like high speed, dynamic changing topology and difficult communication environment. Due to these unique features, routing in vehicular network has always been a challenging issue but besides efficient routing very less attention is being paid in the area of load balancing. So in this study, load optimization/management in VANET is focused and a new protocol is introduced with new metric i.e. local delay which uses interface queue length. The new protocol is an extension of conventional AODV and it is modified according to VANET parameters. The protocol is simulated on Ns2.34 and the performance analysis shows that the new protocol is better than AODV in terms of PDR and EED.
\end{abstract}

\section{General Terms}

Vehicular networks, queue length,AODV, stability,load balancing, performance.

\section{Keywords}

VANET,ns2,pdr,nrl,speed,aggregate interface queue length, end to end delay etc.

\section{INTRODUCTION}

Vanet is new communication paradigm that enables the communication platform between high speed vehicles on the roads.VANETs are direct offshoot of MANETs but with distinguishing characteristics like difficult communication environment with short link lifetime, mobility with high speed, unpredictable node density etc.Several routing protocols are available for VANETs but none of them solve the issue of balancing load in the network. One of the purposes of VANET is improving road safety by providing accidental information to vehicles so that drives could take immediate action in time. Although the protocols designed so far works for efficient data delivery but lacks the addressing of balancing load in congested networks. Due to which some nodes/routes gets congested and some remain idle. This results in poor performance of network. Therefore the need of load distribution among nodes becomes important. In this study, issue of managing load in network is considered and to execute this problem AODV is chosen as candidate protocol and modify/extend the basic routing scheme with new metric. The choice of AODV is due to the reason that this is the only routing protocol that initiates route discovery with flooding route request message and creates route on demand but the drawback is that it does not have any provision of describing the quality of route so a metric interface queue length is introduced .This metric distributes the load from heavy node to lighter one. In VANETs links break very often due to very high speed of vehicle and unpredictable density. So much time is wasted in discovering breaks and maintaining the routes. Although AODV has its own way of doing repair but an extension is done with new metric i.e. local delay to find the alternate path for data delivery so that data must be reached to destination anyway without wasting time in repair. The whole study is executed on Ns2.34 as simulation tool. The platform chosen is again an open source and newly introduced operating system UBUNTU 12.04.The performance metrics are PDR-packet delivery ratio, EED-end to end delay,NRL-normalised routing load.

\section{ADHOC ON DEMAND DISTANCE VECTOR ROUTING}

AODV-adhoc on demand distance vector routing is reactive routing protocol. In this routing scheme routes are created ondemand I..e when the source node wants to send data to destination. To execute the load management strategy in vehicular adhoc network,AODV is chosen as base protocol. The complete mechanism is explained below-

\subsection{AODV route discovery}

AODV $[1,2]$ performs route discovery by broadcasting RREQ to all its neighboring nodes. The broadcasted RREQ contains address of source, destination their sequence numbers, broadcast id and a counter which counts how many times RREQ has been generated for a particular node. When a source broadcast a RREQ it acquires a RREP from its neighbors or that neighbors rebroadcast RREQ to their neighbors by incrementing in the hop count. Node drops repeated RREQ to make the communication loop free.

\subsection{AODV route table management}

AODV route table management is needed to avoid those entries of nodes that do not exists in the route from source to destination. Route table management is done with the help of destination sequence numbers.

\subsection{AODV route maintenance}

When a node detects that a route is not valid anymore for communication it deletes all the related entries from the routing table for those invalid routes. It then sends the RREP to current neighboring nodes that route is not valid anymore.

\section{PROPOSED PROTOCOL- LBV_AODV (LOAD BALANCING PROTOCOL FOR VANET USING AODV)}

The new protocol adopts basic AODV mechanism. It extends route discovery and route repair routines of aodv.In route discovery based on aggregate interface queue length, the route is diverted from heavy node to lighter node and in this way 
load is balanced in the network. And in route repair mechanism when the broken link is detected, the path is diverted to alternate route so that data must be reached to the destination anyway. The alternate path is found by calculating local delay.[7..11].The metric local delay diverts the data on lighter route and hence load is balanced again in repairing the link. The complete algorithm is explained below-

\subsection{Algorithm-Route Request}

Letn is the number of vehicles and $\mathrm{N}$ is the set of vehicles I.e. $N=N 1, N 2, N 3 \ldots \ldots \ldots . . . N n)$; where $N s, N d \nsubseteq N$ and $N s \neq N d$.

$N S=$ source node, $N d=$ destination node

=intermediate node

Step 1: initiate Route Discovery i.e. RREQ

Step 2: Repeat through step 4

Step 3: if ( destination)

$$
\text { forj }=2,3 \ldots \ldots n
$$

call RREP

else

if intermediate node does not have route to destination it adds the no of packets in its interface queue and broadcasts it further or if it has route to destination/aggregate interface queue length is smaller, intermediate node replies with the route.

Step 4: update routing table entries.

Step 5: EXIT.

\subsection{Algorithm-Route Reply}

Step 1: repeat through step 2 for $\mathrm{j}=\mathrm{d}$ to $\mathrm{s}$ generate route reply

Step 2: if $(N j \neq N s)$

call RREP

update routing table entries.

Else

EXIT

\subsection{Algorithm-Route Error}

Step 1: initialize threshold $=T h$.

Step 2: if node detects a link break to next node

Step 3: calculate the local delay on alternate path and send the route request on less loaded path. Local delay is calculated asLocalDelay $=$ Ail $/$ Amax

where $A i l$ is no of packets in buffer and Amax is max length of buffer

else if $(T T L<T h$.

call RREQ

else

print( "Error message");

end if

end if

Step 4: Exit.

\section{SIMULATION SETUP PERFORMANCE METRICS}

AND

Simulation is performed on network simulator ns 2.34 which is an discrete event simulator.[3,4,5,6].To measure the performance of proposed protocol the following scenario is created shown in Table1 and the protocol is analysed on three performance metrics-PDR,EED and NRL.

\subsection{Simulation Parameters}

The simulation parameters are summarised in Table 1.

Table 1 Simulation Setup

\begin{tabular}{|l|l|}
\hline Parameters & Values \\
\hline Network simulator & Ns2.34 \\
\hline Channel & Wireless channel \\
\hline Mobility Model & Random way point \\
\hline Mac Layer & Mac/802.11 \\
\hline Interface Queue & Queue/Droptail/Priqueue \\
\hline Link layer & LL \\
\hline Antenna & Antenna/OmniAntenna \\
\hline $\begin{array}{l}\text { X,Ydimension } \\
\text { topography }\end{array}$ & 1000,1000 \\
\hline No of nodes & 70 \\
\hline Simulation time & $600 s$ \\
\hline Protocol & AODV,LBV_AODV \\
\hline
\end{tabular}

\subsection{Performance Metrics}

The protocol is evaluated on three basic performance metrics1. Packet Delivery Ratio-It is defined as ratio of total number of packets received by total no of packets sent. Greater value of PDR means better performance of the network.

2. End-to-end Delay-This is average time taken by data packet to arrive in the destination. It also includes delay caused by route discovery process and queue in data packet transaction.

3.Normalized Routing Load/Overhead-It is defined as number of routing packets transmitted per data packet.

\section{SIMULATION RESULTS ANALYSIS}

Fig. 1 shows the simulation results of 70 nodes moving at a uniform speed of $20 \mathrm{~m} / \mathrm{s}$. The simulation is performed on a scale a of packet delievery ratio vs pause time.It is observed that as the pause time is varied PDR of new protocol i.e. LBV_AODV is also increased and shows approx.20\% improvement than original AODV.

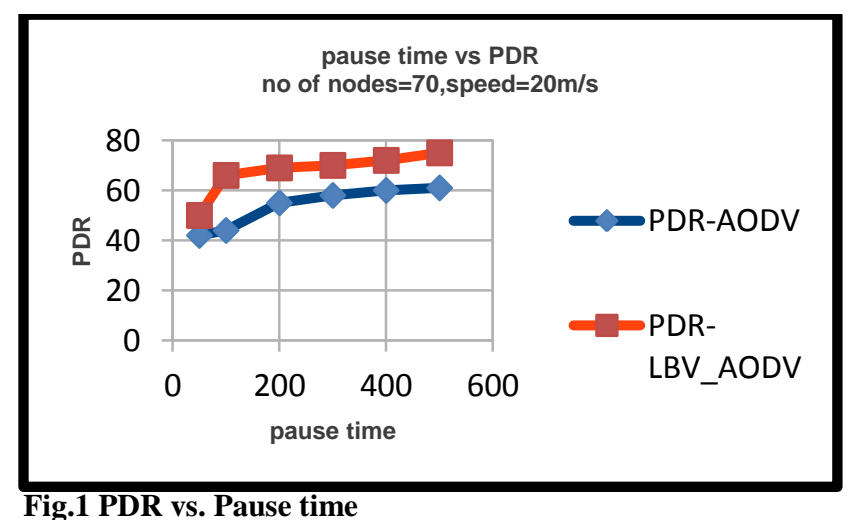

Fig.2 shows performance on a scale of end to end delay vs pause time. It is observed that there is small improvement in delay.EED of new protocol is approximate 6-9\% decreased from original AODV.The graph of EED vs. pause time is shown below- 


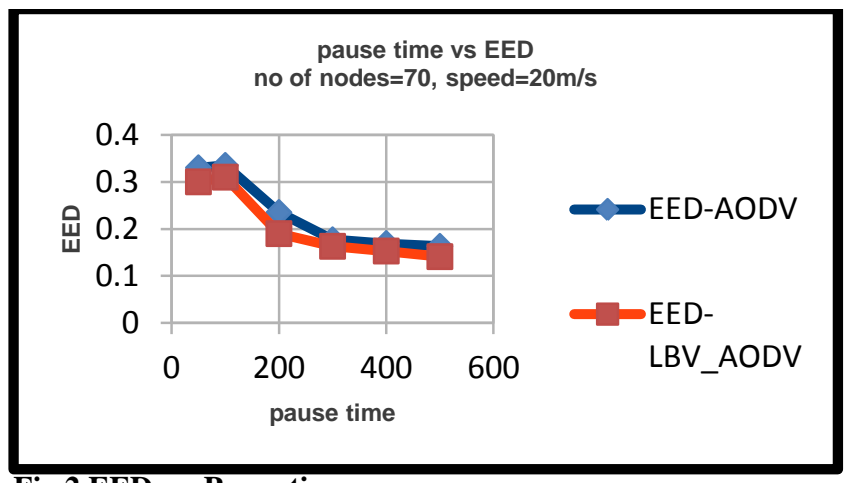

Fig.2 EED vs. Pause time

Fig.3 shows the simulation results of routing overhead/normalized routing load w.r.t. Pause time. It is observed that routing load is increased from previous protocol. This increased NRL is due to the extra overhead taken in repairing the broken link.The graph is shown below-

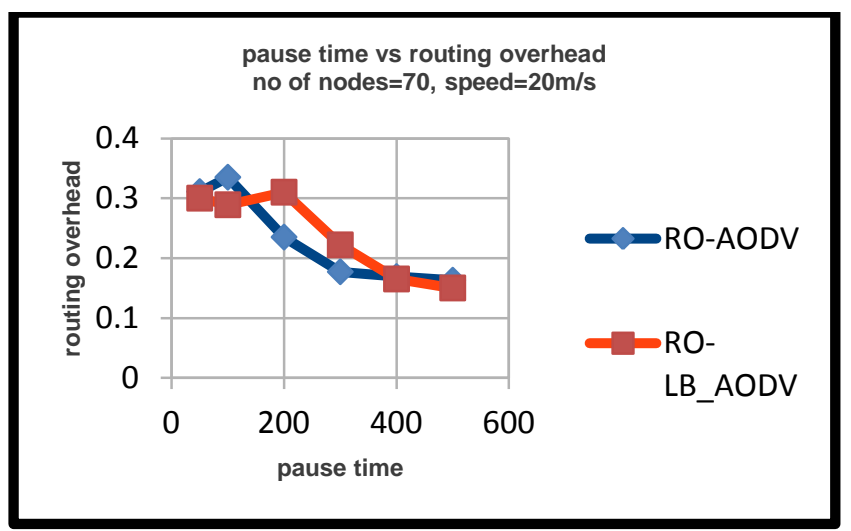

Fig.3 RO vs. Pause time

\section{CONCLUSION}

The load balancing mechanism reduces the load of the network and hence the data is delivered to destination easily and early. Data delivery to destination vehicle in vehicular network is very important because it gives a chance to the driver to take immediate action in case of any accident on road. In this paper the proposed technique is able to balance the load and distributes the message on stable routes. The technique increases the PDR by $18-20 \%$ of original AODV and delay is reduced by $6-9 \%$ however compromising routing overhead. But since the delivery of data is more important in vehicular networks to provide road safety, it is concluded that the new protocol is suitable for VANET.

\section{REFERENCES}

[1] C.Perkins,S.Das 2003, Adhoc on demand distance vector routing,RFC 3561IETF,http://www.ietf.org/rfc3561.txt

[2] C.liu,J.Kaiser,A survey of mobile adhoc network routing protocols, university of Ulm technical report series,No.2003-08,germany 2005.

[3] Fan li,Yu Wang,Routing in vehicular adhoc networks:A survey,IEEE vehicular technology magazine,june 2007.

[4] Marc Greis's Tutorial for UCB/LBNL/VINT Network Simulator "Ns", http://www.isi.edu/nsnam/ns/tutorial

[5] Bijan paul,Md Ibrahim,Md.N.B,Performance evaluation of AODV and DSR with varying pause time and node densityover TCP and CBR connection in vanet,IJCSNS, vol11,issue7,july2011,pg.119-127.

[6] R.A. Santos, A. Edwards, R. Edwards, and L. Seed,Performance evaluation of routing protocols in vehicular adhoc networks, The International Journal of Ad Hoc and Ubiquitous Computing, vol. 1, no. 1/2, pp. 80-91, 2005.

[7] Ben Ding,Z.Chen,H.Yu,An improved AODV routing protocol for VANET,ieeeexplore.ieee.org

[8] A.rani,M.dave,Performance evaluation of modified AODV for load balancing,journal of computer science,ISSN1549-3636.

[9] .A.Dahiya,R.K.Chauhan,A Comparartive study of Manet and Vanet environment,http://www.scribd.com/doc/34832829/AComparative-study-of-MANET-and-VANETEnvironment

[10] M.C. Schut et al, SOTRIP: A self organizing protocol for traffic information,IWCMC, pp. 21-24, 2009.

[11] Car2Car communication consortium,http:Nwww.car-2car.org. 